\title{
A new cubic simplified perturbed hard-body equation of state
}

\author{
Shi-Fang Chen, Yo-Li Chou, Yan-Ping Chen * \\ Department of Chemical Engineering, National Taiwan University, Taipei, Republic of China \\ Received 10 February 1995; accepted 17 August 1995
}

\begin{abstract}
A new cubic equation of state (EOS) was developed in this study for vapor-liquid equilibrium (VLE) calculations of nonpolar fluids. The repulsive term of this EOS reexpressed the results of Walsh and Gubbins (1990) from their modified thermodynamic perturbation theory of polymerization into a simple form in which a non-spherical parameter was employed to account for the different shapes of molecules. The repulsive compressibility factors calculated from this EOS agree well with the molecular simulation data for various kinds of hard bodies ranging from a single hard sphere to tangent or fused long chain molecules. A simple attractive term was then coupled with the repulsive to complete the EOS in a cubic form. Equation parameters were determined for a diversity of nonpolar real fluids. These parameters were expressed in generalized forms for engineering computations. Satisfactory results from this EOS on the saturated properties of pure nonpolar fluids were obtained. This EOS was also extended to calculate the VLE of nonpolar fluid mixtures. The results are again satisfactory over wide ranges of temperature and pressure.
\end{abstract}

Keywords: Theory; Equation of state; Cubic; Perturbed hard body; Vapor-liquid equilibria; Nonpolar fluids

\section{Introduction}

Equations of state (EOS) have been extensively used in phase equilibrium calculations for engineering separation processes. To improve their consistency with the molecular simulation results, various theoretically-based EOS for hard-body fluids have been developed in recent years. Examples include the recent EOS of Boublik et al. (1990) for chain molecules, the new analytical EOS of Song and Mason (1990) for hard convex molecules and the Carnahan-Starling equation for hard spheres (1969). A number of EOS have been constructed by simply incorporating these hard-body equations with contributions of the attractive interactions, according to the perturbation theory. However, most of these perturbed EOS are rather complex. Several authors have further simplified the repulsive

\footnotetext{
${ }^{*}$ Corresponding author.
} 
Table 1

Simplified hard-body equations of state

Lin et al. (1983)

$\mathrm{Z}=\frac{V+0.77 b}{V-0.42 b}$

Soave (1990)

$\mathrm{Z}=\frac{V+(c-1) b}{V-b}$

Elliott et al. (1990)

$\mathrm{Z}=\frac{V+(c-0.475) b}{V-0.475 b}$

Wang and Guo (1993)

$\mathrm{Z}=\frac{V+0.66830 b}{V-0.44744 b}$

Shah et al. (1994)

$\mathrm{Z}=\frac{V}{V-k_{0} \beta}+\frac{\beta k_{1} V}{\left(V-k_{0} \beta\right)^{2}}$

$\mathrm{k}_{0}=1.2864 ; \mathrm{k}_{1}=2.8225$

equations for hard spheres in association with a simple attractive terms as perturbation to formulate equations of state that are in a cubic or quartic form without loss of their theoretical basis (Lin et al., 1983; Wang and Guo, 1993; Shah et al., 1994). Some of the simplified forms for the repulsive compressibility factor are listed in Table 1.

Real molecules are not spherically symmetric except for few simple fluids. It is of interest to consider a parameter in the repulsive contribution of an EOS due to different shapes and geometries of molecules. Elliott et al. (1990) presented an equation for nonspherical and associating fluids which included a shape parameter $\mathrm{c}$ to describe different hard convex bodies and tangent long chain molecules, while Soave (1990) introduced a c-parameter, which is dependent on molecular dimensions, to express the repulsive contribution. Many theories have also been proposed to represent the thermodynamic properties of different hard molecules. For example, the modified thermodynamic perturbation theory (MTPT) of polymerization (Walsh and Gubbins, 1990) has been applied to describe the properties of various types of hard bodies with satisfactory results (Thomas and Donohue, 1993).

In this work, we presented a new simplified form of the Walsh-Gubbins equation for hard molecules of various shapes and geometries. The calculated repulsive compressibility factors from this equation were compared with the computer simulation data in literature. An empirical form for the attractive part was also proposed in perturbation around this reference repulsive term to complete a cubic EOS. Equation parameters for nonpolar real molecules were determined from the saturated properties of pure fluids. The parameters were correlated in generalized forms. A comparison of the new equation with other cubic EOS in the representation of PVT was presented. This EOS was further 
employed to calculate the vapor-liquid equilibria (VLE) of nonpolar mixtures. The calculated results were also compared with those from other commonly-used cubic EOS.

\section{Development of the equation of state}

This study proposed a simplified form for the expression of the repulsive contribution of an EOS. According to the hard-body EOS of Walsh and Gubbins (1990), the repulsive compressibility factor was written as:

$$
z^{\text {rep }}=1+(2 \alpha-1) \frac{4 \eta-2 \eta^{2}}{(1-\eta)^{3}}+2(\alpha-1)\left(\frac{\eta}{2-\eta}-\frac{3 \eta}{1-\eta}\right)
$$

where $\alpha$ is the nonspherical factor and $\eta$ the packing fraction. This equation describes hard molecules of various geometries with specific $\alpha$ values and reduces to the Carnahan-Starling EOS for hard spheres (Carnahan and Starling, 1969) as $\alpha=1$. The Walsh-Gubbins EOS is simplified into the following form:

$$
z^{\text {rep }}=\frac{V+K_{1} b}{V-K_{2} b}
$$

where $b$ represents the hard core molar volume and hence $b / V$ is the packing fraction. $K_{1}$ and $K_{2}$ are adjustable parameters, which are determined by fitting the equation to Eq. (1) over the range of packing fraction up to 0.5 , and the range of the nonspherical factor, $\alpha$, from a single hard sphere $(\alpha=1)$ to hard convex bodies of different shapes and chainlike molecules of 201 tangent spheres. The values were correlated as a function of the nonspherical factor $\alpha$ as will be discussed later.

This study further suggests a simple expression for the attractive term to incorporate with Eq. (2) to form the following EOS:

$$
P=\frac{R T\left(1+K_{1} b / V\right)}{V-K_{2} b}-\frac{a}{V(V+c)}
$$

Eq. (3) is explicit in pressure and third-order (cubic) in volume. By employing the critical constraints:

$$
\left(\frac{\partial P}{\partial V}\right)_{c}=\left(\frac{\partial^{2} P}{\partial V^{2}}\right)_{c}=0
$$

and

$$
\frac{P_{\mathrm{c}} V_{\mathrm{c}}}{R T_{\mathrm{c}}}=\zeta_{\mathrm{c}}
$$

Equation parameters $\mathrm{a}, \mathrm{b}$ and $\mathrm{c}$ can be expressed in terms of the critical constants as:

$$
\begin{aligned}
& a=\Omega_{\mathrm{ac}} A(T) R^{2} T_{\mathrm{c}}^{2} / P_{\mathrm{c}} \\
& b=\Omega_{\mathrm{bc}} B(T) R T_{\mathrm{c}} / P_{\mathrm{c}}
\end{aligned}
$$


and

$$
c=\Omega_{\mathrm{cc}} R T_{\mathrm{c}} / P_{\mathrm{c}}
$$

where $A(T)$ and $B(T)$ are temperature-dependent functions and $\Omega_{\mathrm{ac}}, \Omega_{\mathrm{bc}}$ and $\Omega_{\mathrm{cc}}$ are constants. $\Omega_{\mathrm{bc}}$ is the smallest positive root of the following equation:

$$
\begin{aligned}
& K_{2}^{3} \Omega_{\mathrm{bc}}^{3}+\left(2 K_{1} K_{2}+2 K_{2}^{2}-3 K_{2}^{2} \zeta_{\mathrm{c}}\right) \Omega_{\mathrm{bc}}^{2} \\
& \quad+\left(K_{1}+K_{2}-3 K_{1} \zeta_{\mathrm{c}}-3 K_{2} \zeta_{\mathrm{c}}+3 K_{2} \zeta_{\mathrm{c}}{ }^{2}\right) \Omega_{\mathrm{bc}}-\zeta_{\mathrm{c}}{ }^{3}=0
\end{aligned}
$$

and

$$
\begin{aligned}
& \Omega_{\mathrm{cc}}=1+K_{2} \Omega_{\mathrm{bc}}-3 \zeta_{\mathrm{c}} \\
& \Omega_{\mathrm{ac}}=\left(\zeta_{\mathrm{c}}{ }^{3}-K_{1} \Omega_{\mathrm{bc}} \Omega_{\mathrm{cc}}\right) / K_{2} \Omega_{\mathrm{bc}}
\end{aligned}
$$

To evaluate the EOS parameters for a pure real molecule, the critical constants (temperature, pressure, compressibility factor) and the nonspherical factor $\alpha$ are required. The temperature-dependent functions $A(T)$ and $B(T)$ were determined by imposing the equal fugacity criterion along each isotherm of the saturation curve:

$$
f^{\mathrm{V}}=f^{\mathrm{L}}
$$

where

$$
\begin{aligned}
& \ln \frac{f}{P}=z-1-\ln z+\frac{K_{1}+K_{2}}{K_{2}} \ln \frac{z}{z-K_{2} B}+\frac{A}{C} \ln \frac{z}{z+C} \\
& A=P a / R^{2} T^{2} \\
& B=P b / R T
\end{aligned}
$$

and

$$
C=P c / R T
$$

The regression was based on the following objective function to minimize the deviations in vapor pressure and saturated liquid volume:

$$
Q=\Sigma\left[\left|\frac{P^{\text {vap,cal }}-P^{\text {vap,exp }}}{P^{\text {vap,exp }}}\right|+\left|\frac{V^{\mathrm{L}, \text { cal }}-V^{\mathrm{L}, \text { exp }}}{V^{\mathrm{L}, \exp }}\right|\right]
$$

where the summation is over the saturation curve.

This EOS was also used for VLE calculations of nonpolar mixtures. In the mixture calculations, the following mixing rules were employed to evaluate the EOS mixture parameters from the properties of constituent components:

$$
\begin{aligned}
& \alpha_{\mathrm{m}}=\sum_{\mathrm{i}} x_{\mathrm{i}} \alpha_{\mathrm{i}} \\
& a_{\mathrm{m}}=\sum_{\mathrm{i}} \sum_{\mathrm{j}} x_{\mathrm{i}} x_{\mathrm{j}}\left(a_{\mathrm{i}} a_{\mathrm{j}}\right)^{0.5}\left(1-k_{\mathrm{ij}}\right) \\
& b_{\mathrm{m}}=\sum_{\mathrm{i}} \sum_{\mathrm{j}} x_{\mathrm{i}} x_{\mathrm{j}}\left[\left(b_{\mathrm{i}}+b_{\mathrm{j}}\right) / 2\right]\left(1-1_{\mathrm{ij}}\right)
\end{aligned}
$$


and

$$
c_{\mathrm{m}}=\sum_{\mathrm{i}} x_{\mathrm{i}} c_{\mathrm{i}}
$$

where $k_{\mathrm{ij}}$ and $l_{\mathrm{ij}}$ are the two binary interaction parameters that were determined by minimizing the following objective function in the bubble-point pressure calculations:

$$
Q^{\prime}=\sum\left[\left|\left(P^{\text {cal }}-P^{\exp }\right) / P^{\exp }\right|+\mid y^{\text {cal }}-y^{\exp \mid}\right]
$$

For the VLE calculations, fugacities of component $i$ in the equilibrium vapor and liquid phases are equal:

$$
\hat{f}_{\mathrm{i}}^{\mathrm{V}}=\hat{f}_{\mathrm{i}}^{\mathrm{L}}
$$

The fugacity coefficient of component $\mathrm{i}$ from this EOS is expressed as:

$$
\begin{aligned}
& \ln \hat{\phi}_{\mathrm{i}}=\ln \frac{V}{V-K_{2} b_{\mathrm{m}}}-\frac{a_{\mathrm{m}} \hat{c}_{\mathrm{i}}}{R T\left(c_{\mathrm{m}}^{2}+c_{\mathrm{m}} V\right)}+\frac{1}{R T}\left[\frac{\hat{a}_{\mathrm{i}}}{c_{\mathrm{m}}}-\frac{a_{\mathrm{m}} \hat{c}_{\mathrm{i}}}{c_{\mathrm{m}}^{2}}\right] \ln \left(\frac{V}{V+c_{\mathrm{m}}}\right) \\
& +\frac{\left(K_{1}+K_{2}\right)\left[K_{2} \hat{b}_{\mathrm{i}}+n b_{\mathrm{m}}\left(\partial K_{2} / \partial n_{\mathrm{i}}\right)\right]}{K_{2} V-K_{2}^{2} b_{\mathrm{m}}}-\ln z \\
& +\frac{\left[K_{1} K_{2}+n K_{2}\left(\partial K_{1} / \partial n_{\mathrm{i}}\right)-n K_{1}\left(\partial K_{2} / \partial n_{\mathrm{i}}\right)\right]}{K_{2}^{2}} \ln \left(\frac{V}{V-K_{2} b_{\mathrm{m}}}\right)
\end{aligned}
$$

where

$$
\begin{aligned}
& \hat{a}_{\mathrm{i}}=n^{-1}\left(\partial n^{2} a_{\mathrm{m}} / \partial n_{\mathrm{i}}\right)_{T, V, n_{\mathrm{j} \neq \mathrm{i}}} \\
& \hat{b}_{\mathrm{i}}=\left(\partial n b_{\mathrm{m}} / \partial n_{\mathrm{i}}\right)_{\mathrm{T}, \mathrm{V}, \mathrm{n}_{\mathrm{j} \neq \mathrm{i}}}
\end{aligned}
$$

and

$$
\hat{c}_{\mathrm{i}}=\left(\partial n c_{\mathrm{m}} / \partial n_{\mathrm{i}}\right)_{\mathrm{T}, \mathrm{V}, \mathrm{n}_{\mathrm{j} \neq \mathrm{i}}}
$$

The parameters $K_{1}$ and $K_{2}$ were given in Eqs. (28) and (29) as a function of the nonspherical factor $\alpha$. Their derivatives with respect to $n_{\mathrm{i}}$ in Eq. (24) are evaluated by using the correlation equations and the mixing rule of $\alpha_{\mathrm{m}}$.

\section{Results and discussion}

The repulsive term of the EOS has two parameters $K_{1}$ and $K_{2}$, which were determined by directly adjusting to the repulsive compressibility factors from Eq. (1) at various $\alpha$ values over the packing fractions up to 0.5 . The resulting values were correlated in generalized forms as a function of the nonspherical factor $\alpha$ :

$$
K_{1}=4.8319 \alpha-1.5515
$$

and

$$
K_{2}=1.8177-0.1778 \alpha^{-1.3686}
$$




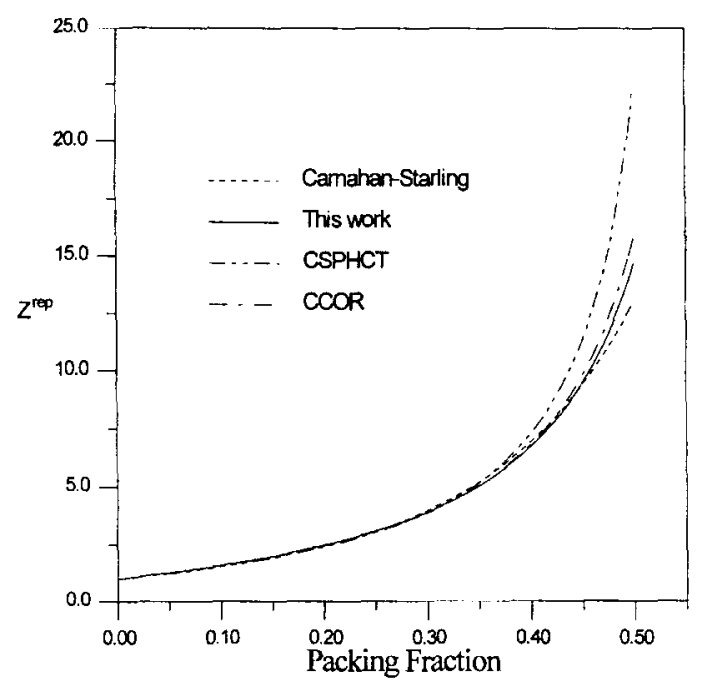

Fig. 1. Comparison of the compressibility factors of hard spheres calculated from various equations of state.

When the $\alpha$ value is unity, Eq. (2) reduces to approximate the hard sphere EOS of Carnahan and Starling. Fig. 1 shows a comparison of the calculated compressibility factors for hard spheres by different EOS. The CCOR EOS (Lin et al., 1983), the CSPHCT EOS (Wang and Guo, 1993) and the EOS of this work all agree well with the Carnahan-Starling EOS at low packing fractions. At higher packing fractions, the CCOR EOS and Eq. (2) of this work yield better results. Eq. (2) was further used to calculate the compressibility factors of nonspherical hard molecules. Some of the calculated results are illustrated in Figs. 2-6. Figs. 2 and 3 show the calculated compressibility factors for tangent chain molecules. Eq. (2), even in a simplified form, appears to agree satisfactorily with the molecular simulated data (Dickman and Hall, 1988; Denlinger and Hall, 1990; Gao and Weiner,

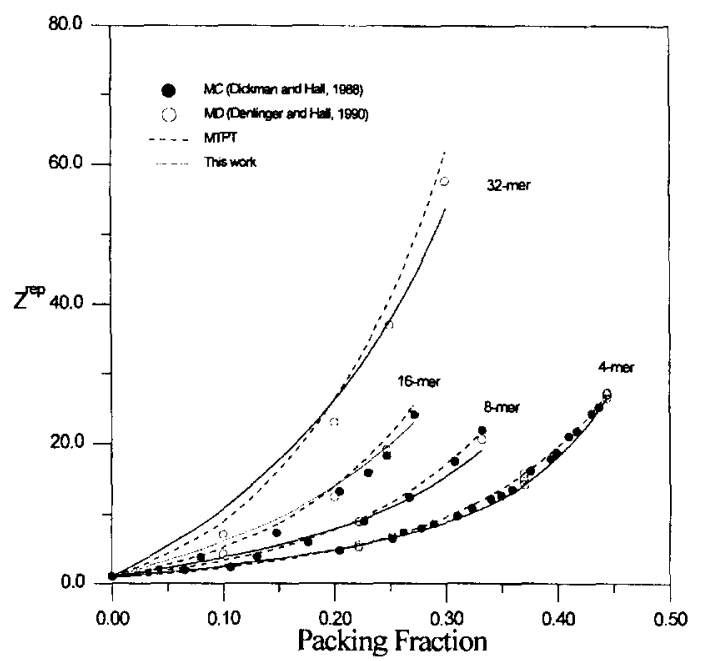

Fig. 2. Comparison of the calculated compressibility factors of tangent hard sphere chains from this work with those from the MTPT EOS and the simulation data (4-mer to 32-mer). 


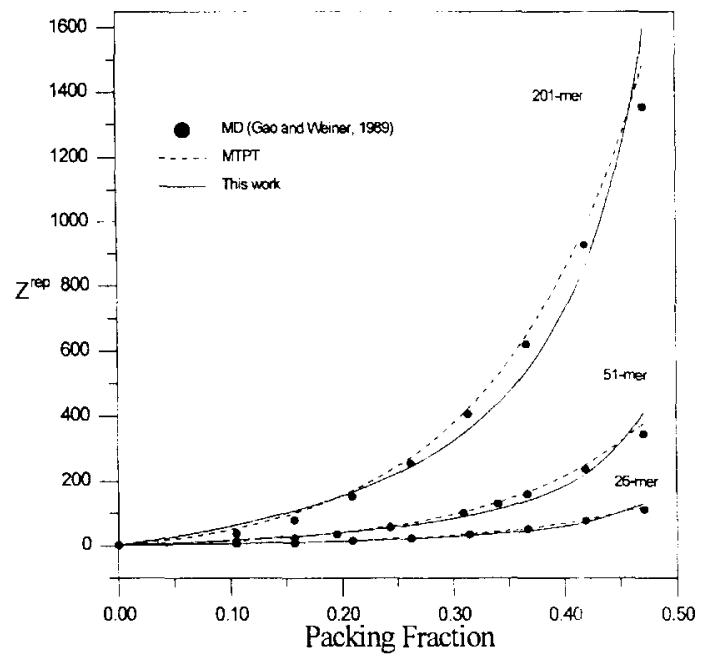

Fig. 3. Comparison of the calculated compressibility factors of tangent hard sphere chains from this work with those from the MTPT EOS and the simulation data (26-mer to 201 -mer).

1989) and with the results calculated from the Walsh-Gubbins EOS over wide ranges of packing fraction and nonspherical factor. Figs. 4-6 show the calculated compressibility factors for hard convex molecules of different shapes and geometries. Generally, the calculated results agree satisfactorily with the molecular simulation data of Boublik and Nezbeda (1986) and compare well with the Walsh-Gubbins equation from the MTPT. Overall 244 data points of different hard-body molecules have been compared. The grand absolute average deviations of the calculated compressibility factors by Eqs. (1) and (2) from the molecular simulation data (Alejandre and Chapela, 1987; Amos and

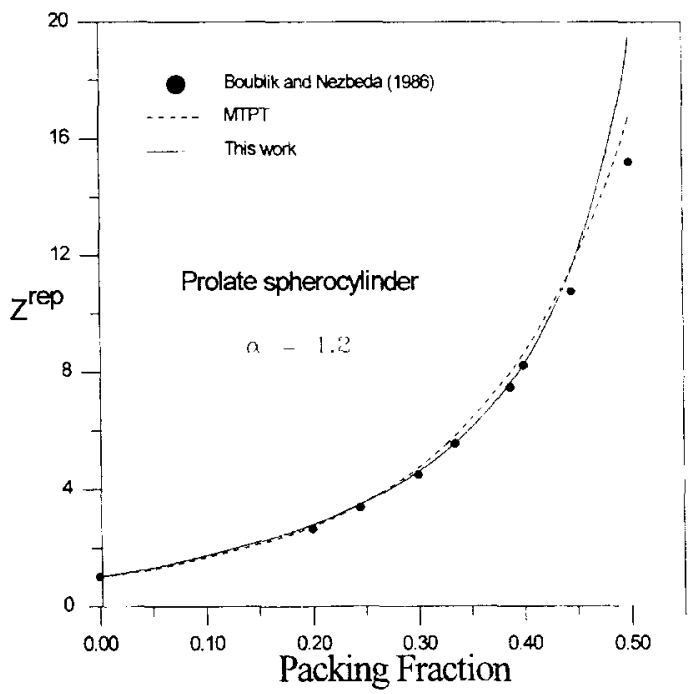

Fig. 4. Compressibility factors of hard prolate spherocylinder calculated using the MTPT EOS and the simplified form of this work (This $\alpha$ corresponds to the ratio of major-to-minor axes of 2). 


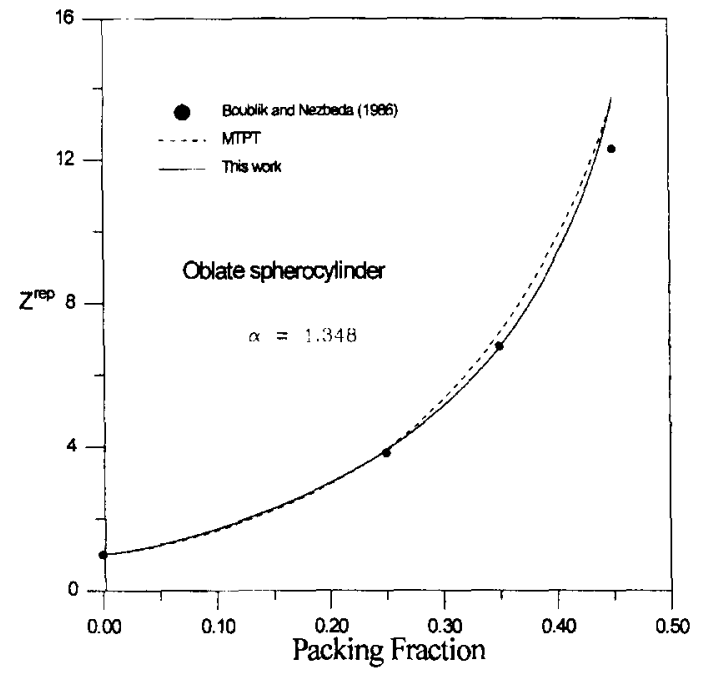

Fig. 5. Compressibility factors of hard oblate spherocylinder calculated using the MTPT EOS and the simplified form of this work (This $\alpha$ corresponds to the ratio of major-to minor axes of 3).

Jackson, 1991; Boublik and Nezbeda, 1986; Boublik et al., 1990; Dickman and Hall, 1988; Denlinger and Hall, 1990; Gao and Weiner, 1989) are 8 and 10\%, respectively. These results indicate that Eq. (2) is comparable with the Walsh-Gubbins EOS but retains the simplicity of its equation form. We thus apply Eq. (2) as a reference for the repulsive contribution to the proposed EOS of Eq. (3).

Eq. (3) was extended to calculate the vapor-liquid equilibria of real nonpolar fluids. Equation parameters can be evaluated by using the following correlations. The critical compressibility factor is given by:

$$
\zeta_{\mathrm{c}}=0.291-0.08 \omega
$$

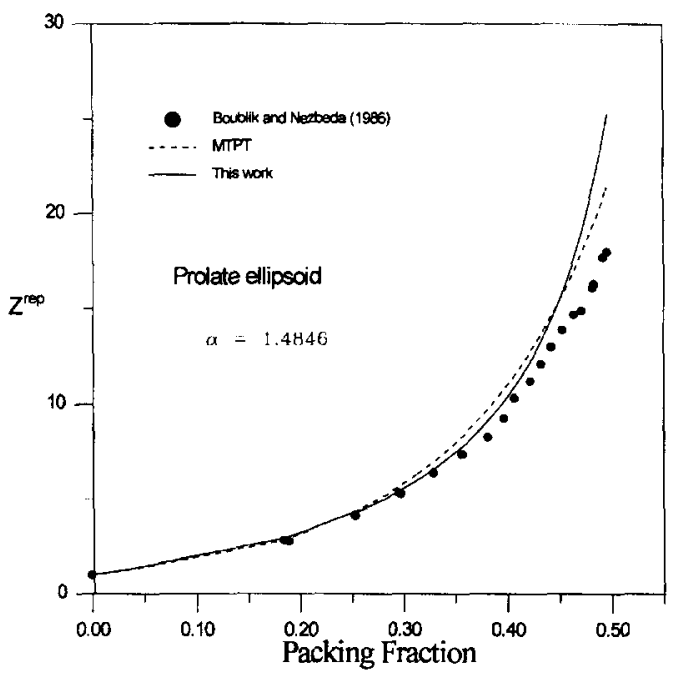

Fig. 6. Compressibility factors of hard prolate ellipsoid calculated using the MTPT EOS and the simplified form of this work (This $\alpha$ corresponds to the ratio of major-to minor axes of 3 ). 


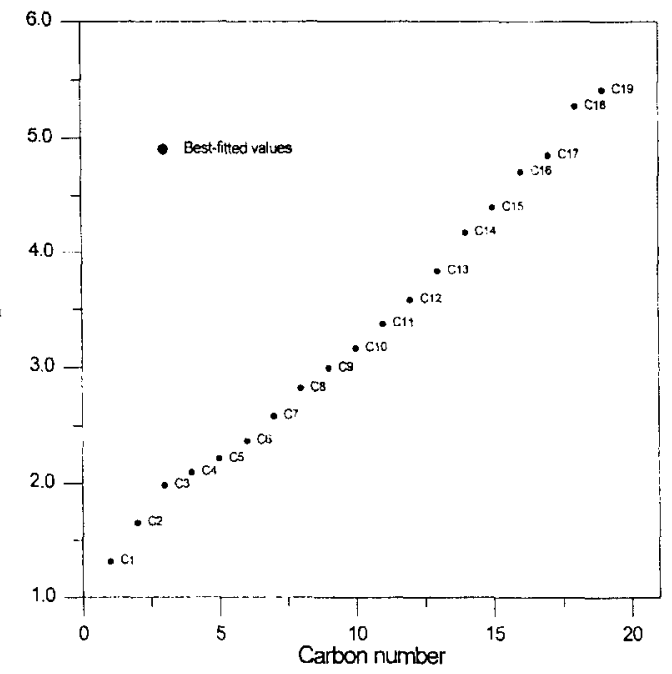

Fig. 7. Best fitted parameters $\alpha$ for the EOS of this work against the carbon numbers of $n$-paraffins.

This correlation is good for hydrocarbons and has been used by other studies in their development of equations of state (e.g., Lin et al., 1983). The temperature-dependent parameters a and b in Eqs. (6) and $(7)$ were found to be well-correlated by the following equations:

$$
A(T)=\left[1+F_{1} \ln T_{\mathrm{r}}+F_{2}\left(1-\sqrt{T_{\mathrm{r}}}\right)^{2}\right]^{2}
$$

and

$$
B(T)=\left[1+F_{3} \ln A(T)\right]^{2}
$$

where

$$
\begin{aligned}
& F_{1}=-0.6525-2.1911 \omega+4.7537 \omega^{2}-11.3392 \omega^{3}+8.6979 \omega^{4} \\
& F_{2}=-2.1278-7.8507 \omega+29.846 \omega^{2}-74.902 \omega^{3}+59.089 \omega^{4}
\end{aligned}
$$

and

$$
F_{3}=1.0542+0.4905 \omega-0.8984 \omega^{2}+4.0568 \omega^{3}-2.9448 \omega^{4}
$$

The nonspherical factor, $\alpha$, can be evaluated from the size and shape of a molecule. However, in practical calculations, its value was usually obtained by adjusting the equation to the phase equilibrium data of real fluids. Fig. 7 shows a plot of the best-fitted values of $\alpha$ versus carbon numbers of $n$-paraffins. These optimal values of $\alpha$ apparently increase with the molecular dimensions, consisting with its physical significance. The $\alpha$ factor can also be generalized in terms of acentric factor:

$$
\alpha=1.2946+4.5671 \omega-4.6784 \omega^{2}+6.2596 \omega^{3}
$$

Phase equilibrium calculations of this work for both pure fluids and their mixtures were based on these generalized correlations for the EOS parameters. Table 2 presents the calculated results on the 
Table 2

Comparison of saturated vapor pressures and liquid volumes of pure substances calculated from various cubic equations of state

\begin{tabular}{|c|c|c|c|c|c|c|c|c|c|c|c|c|c|}
\hline \multirow[t]{2}{*}{ Compound } & \multirow{2}{*}{$\begin{array}{l}\text { Temp. } \\
\text { range } \\
(\mathrm{K})\end{array}$} & \multicolumn{2}{|c|}{$\begin{array}{l}\text { No. of data } \\
\text { points }\end{array}$} & \multicolumn{5}{|c|}{$\operatorname{AADP}(\%)$} & \multicolumn{5}{|c|}{$\operatorname{AADV}^{L}(\%)$} \\
\hline & & $P^{\text {sat }}$ & $V^{\mathrm{L}}$ & PR & PT & YL & CCOR & $\begin{array}{l}\text { This } \\
\text { work }\end{array}$ & PR & PT & YL & CCOR & $\begin{array}{l}\text { This } \\
\text { work }\end{array}$ \\
\hline Argon & $84-149$ & 68 & 65 & 2.24 & 2.82 & 1.54 & 2.20 & 1.40 & 10.82 & 4.03 & 4.63 & 2.76 & 2.55 \\
\hline Krypton & $116-208$ & 47 & 46 & 0.66 & 0.87 & 1.31 & 0.98 & 1.33 & 7.75 & 4.36 & 2.65 & 1.49 & 1.63 \\
\hline Oxygen & $66-152$ & 88 & 88 & 0.75 & 1.17 & 1.30 & 2.07 & 1.09 & 9.73 & 4.01 & 3.56 & 1.66 & 0.82 \\
\hline Nitrogen & $63-125$ & 64 & 64 & 0.43 & 0.58 & 1.43 & 1.04 & 0.51 & 8.66 & 4.47 & 3.56 & 1.73 & 0.82 \\
\hline Carbon Dioxide & $217-302$ & 42 & 42 & 1.05 & 0.96 & 1.96 & 0.97 & 1.06 & 6.02 & 7.23 & 6.04 & 2.16 & 1.51 \\
\hline Methane & $91-187$ & 44 & 44 & 0.69 & 1.27 & 0.84 & 1.29 & 0.63 & 8.11 & 4.24 & 2.59 & 1.54 & 1.88 \\
\hline Ethane & $163-300$ & 29 & 29 & 0.44 & 0.91 & 1.12 & 1.32 & 0.28 & 6.07 & 4.01 & 2.50 & 1.19 & 2.37 \\
\hline Propane & $173-363$ & 31 & 31 & 1.91 & 1.51 & 2.31 & 1.40 & 1.05 & 5.46 & 3.49 & 2.31 & 1.25 & 1.65 \\
\hline Butane & $217-392$ & 28 & 28 & 0.86 & 0.68 & 1.34 & 2.19 & 0.33 & 3.87 & 2.19 & 1.50 & 1.17 & 0.41 \\
\hline isoButane & $187-401$ & 34 & 25 & 2.53 & 2.15 & 2.72 & 1.55 & 1.40 & 4.60 & 2.05 & 1.54 & 1.39 & 0.22 \\
\hline Pentane & $240-454$ & 30 & 30 & 0.76 & 0.59 & 0.86 & 1.97 & 0.25 & 3.33 & 2.89 & 2.27 & 1.39 & 0.56 \\
\hline 2-Methylbutane & $236-447$ & 29 & 29 & 0.32 & 0.30 & 0.76 & 1.99 & 0.44 & 4.78 & 3.98 & 3.23 & 2.37 & 1.34 \\
\hline $\begin{array}{l}\text { 2,2-Dimethyl- } \\
\text { propane }\end{array}$ & $257-433$ & 45 & 43 & 0.44 & 0.40 & 1.18 & 1.20 & 0.29 & 5.41 & 4.29 & 3.54 & 2.53 & 1.44 \\
\hline Hexane & $267-492$ & 31 & 31 & 1.02 & 0.96 & 0.54 & 2.26 & 0.56 & 3.08 & 3.06 & 2.77 & 1.77 & 0.74 \\
\hline 2-Methylpentane & $289-497$ & 25 & 23 & 0.36 & 0.39 & 0.65 & 1.31 & 0.11 & 3.35 & 3.19 & 2.77 & 1.86 & 0.89 \\
\hline 3-Methylpentane & $283-504$ & 43 & 43 & 0.66 & 0.59 & 0.68 & 1.54 & 0.41 & 3.90 & 3.65 & 3.13 & 2.11 & 1.10 \\
\hline 2,2-Diethylbutane & $236-488$ & 41 & 31 & 1.06 & 0.78 & 1.32 & 1.59 & 0.31 & 3.85 & 2.99 & 2.37 & 1.87 & 0.85 \\
\hline 2,3-Diethylbutane & $259-499$ & 45 & 40 & 0.56 & 0.49 & 0.99 & 1.61 & 0.23 & 4.19 & 3.73 & 3.10 & 2.11 & 1.10 \\
\hline Heptane & $280-507$ & 29 & 29 & 1.38 & 1.17 & 0.51 & 2.20 & 0.54 & 2.60 & 1.88 & 1.98 & 1.29 & 0.30 \\
\hline 2-Methylhexane & $284-524$ & 41 & 40 & 0.85 & 0.80 & 0.80 & 1.37 & 0.36 & 2.93 & 3.06 & 2.91 & 2.18 & 1.08 \\
\hline 3-Methylhexane & $284-529$ & 44 & 42 & 0.84 & 0.79 & 0.88 & 1.39 & 0.40 & 4.08 & 4.04 & 3.97 & 2.30 & 1.44 \\
\hline 3-Ethylpentane & $300-532$ & 28 & 27 & 0.58 & 0.59 & 0.69 & 1.38 & 0.17 & 3.10 & 3.14 & 2.85 & 2.56 & 1.60 \\
\hline $\begin{array}{l}\text { 2,2-Dimethyl- } \\
\text { pentane }\end{array}$ & $286-512$ & 28 & 27 & 0.65 & 0.57 & 0.94 & 1.32 & 0.26 & 3.83 & 3.67 & 3.27 & 2.47 & 1.47 \\
\hline $\begin{array}{l}\text { 2,3-Dimethyl- } \\
\text { pentane }\end{array}$ & $260-529$ & 37 & 31 & 1.50 & 1.21 & 1.36 & 1.56 & 0.53 & 3.67 & 3.55 & 3.19 & 2.69 & 1.73 \\
\hline $\begin{array}{l}\text { 2,2,3-Trimeth- } \\
\text { ylbutane }\end{array}$ & $273-525$ & 41 & 42 & 0.60 & 0.51 & 0.87 & 1.60 & 0.23 & 4.37 & 3.70 & 3.11 & 2.61 & 1.58 \\
\hline Octane & $312-544$ & 30 & 30 & 1.07 & 1.20 & 0.51 & 1.50 & 0.51 & 4.65 & 2.18 & 2.58 & 1.33 & 0.56 \\
\hline $\begin{array}{l}2,2,4-\text { Triethyl- } \\
\text { butane }\end{array}$ & $291-535$ & 30 & 29 & 1.00 & 1.00 & 0.82 & 2.01 & 0.42 & 3.69 & 3.66 & 3.32 & 3.10 & 2.14 \\
\hline Nonane & $312-503$ & 36 & 12 & 1.22 & 1.41 & 1.37 & 1.41 & 0.38 & 3.86 & 0.68 & 1.02 & 1.81 & 0.62 \\
\hline n-Decane & $331-523$ & 35 & 12 & 2.38 & 1.24 & 1.97 & 0.84 & 1.09 & 4.89 & 0.85 & 1.91 & 2.07 & 0.73 \\
\hline n-Undecane & $348-499$ & 30 & 13 & 2.34 & 1.41 & 1.92 & 1.78 & 0.58 & 5.80 & 1.21 & 2.87 & 2.27 & 0.76 \\
\hline n-Dodecane & $365-520$ & 30 & 12 & 2.40 & 1.86 & 1.31 & 2.73 & 0.64 & 7.90 & 0.74 & 2.69 & 1.10 & 1.85 \\
\hline n-Tridecane & $380-540$ & 30 & 13 & 3.27 & 1.47 & 0.50 & 3.35 & 0.43 & 7.64 & 1.75 & 4.72 & 1.92 & 0.48 \\
\hline n-Tetradecane & $395-559$ & 30 & 12 & 4.14 & 0.99 & 0.82 & 4.48 & 0.54 & 8.08 & 2.59 & 6.55 & 1.89 & 0.99 \\
\hline n-Pentadecane & $409-577$ & 30 & 12 & 4.69 & 1.32 & 0.44 & 5.78 & 0.56 & 9.44 & 2.36 & 7.12 & 0.69 & 1.72 \\
\hline n-Hexadecane & $422-594$ & 30 & 11 & 2.23 & 3.02 & 2.90 & 9.77 & 2.58 & 12.44 & 0.83 & 6.38 & 2.25 & 1.45 \\
\hline n-Heptadecane & $435-609$ & 30 & 11 & 5.65 & 2.11 & 1.20 & 8.15 & 1.12 & 16.3 & 2.07 & 4.42 & 6.06 & 0.37 \\
\hline n-Octadecane & $447-624$ & 30 & 10 & 3.00 & 5.31 & 5.10 & 13.30 & 3.28 & 20.78 & 5.48 & 1.81 & 10.5 & 1.51 \\
\hline n-Nonadecane & $458-638$ & 30 & 10 & 4.36 & 3.81 & 3.67 & 12.39 & 1.74 & 24.78 & 7.96 & 0.55 & 14.91 & 0.50 \\
\hline
\end{tabular}


Table 2 (continued)

\begin{tabular}{|c|c|c|c|c|c|c|c|c|c|c|c|c|c|}
\hline \multirow[t]{2}{*}{ Compound } & \multirow{2}{*}{$\begin{array}{l}\text { Temp. } \\
\text { range } \\
(\mathrm{K})\end{array}$} & \multicolumn{2}{|c|}{$\begin{array}{l}\text { No. of data } \\
\text { points }\end{array}$} & \multicolumn{5}{|c|}{$\operatorname{AADP}(\%)$} & \multicolumn{5}{|c|}{$\operatorname{AADV}^{\mathrm{L}}(\%)$} \\
\hline & & $P^{\text {sat }}$ & $\overline{V^{L}}$ & PR & PT & YL & $\mathrm{CCOR}$ & $\begin{array}{l}\text { This } \\
\text { work }\end{array}$ & PR & PT & $\mathrm{YL}$ & $\mathrm{CCOR}$ & $\begin{array}{l}\text { This } \\
\text { work }\end{array}$ \\
\hline Benzene & $284-550$ & 42 & 42 & 1.61 & 1.38 & 2.02 & 1.11 & 0.99 & 3.43 & 3.21 & 2.73 & 1.22 & 2.18 \\
\hline Toluene & $282-546$ & 29 & 29 & 1.10 & 0.85 & 0.58 & 2.69 & 0.67 & 2.62 & 1.80 & 1.32 & 1.77 & 0.74 \\
\hline o-Xylene & $303-630$ & 38 & 36 & 0.76 & 0.75 & 0.49 & 2.75 & 0.82 & 2.90 & 2.80 & 2.97 & 1.19 & 1.14 \\
\hline m-Xylene & $302-616$ & 37 & 33 & 0.47 & 0.70 & 0.61 & 2.67 & 0.82 & 4.26 & 3.60 & 4.02 & 1.60 & 2.64 \\
\hline p-Xylene & $296-616$ & 42 & 26 & 0.59 & 0.83 & 0.73 & 3.02 & 1.10 & 2.72 & 2.23 & 2.70 & 1.30 & 2.75 \\
\hline Ethylbenzene & $297-616$ & 33 & 20 & 1.17 & 0.97 & 0.79 & 1.95 & 0.13 & 0.83 & 0.92 & 1.42 & 0.16 & 1.53 \\
\hline Cyclopropane & $174-397$ & 40 & 34 & 2.12 & 1.74 & 2.29 & 1.99 & 1.15 & 4.95 & 4.27 & 2.59 & 2.06 & 3.47 \\
\hline Cyclobutane & $203-285$ & 41 & 35 & 0.15 & 1.07 & 1.39 & 6.52 & 3.11 & 6.68 & 2.89 & 1.89 & 2.26 & 0.82 \\
\hline Cyclopentane & $229-510$ & 40 & 39 & 2.15 & 1.74 & 2.17 & 1.24 & 0.90 & 5.19 & 3.50 & 2.60 & 1.97 & 0.76 \\
\hline Cyclohexane & $279-491$ & 35 & 35 & 1.87 & 1.51 & 2.31 & 1.10 & 1.09 & 4.02 & 1.76 & 1.30 & 1.57 & 0.43 \\
\hline Cycloheptane & $283-604$ & 45 & 40 & 1.84 & 1.41 & 1.94 & 1.34 & 0.75 & 4.51 & 3.45 & 2.87 & 2.81 & 1.74 \\
\hline Ethene & $120-281$ & 41 & 40 & 2.17 & 1.94 & 2.56 & 1.88 & 1.13 & 6.43 & 3.24 & 1.95 & 1.20 & 2.51 \\
\hline Propene & $163-364$ & 33 & 33 & 1.59 & 1.43 & 1.92 & 1.28 & 0.67 & 6.40 & 3.67 & 2.72 & 1.73 & 0.75 \\
\hline 1-Butene & $215-418$ & 41 & 35 & 0.46 & 0.36 & 0.92 & 2.07 & 0.45 & 3.93 & 1.80 & 1.22 & 0.96 & 0.58 \\
\hline 1-Pentene & $221-463$ & 37 & 34 & 1.37 & 1.02 & 1.58 & 1.59 & 0.35 & 2.66 & 2.35 & 2.23 & 0.76 & 1.68 \\
\hline 1-Hexene & $283-353$ & 45 & 33 & 1.15 & 0.66 & 1.14 & 1.8 & 0.76 & 0.46 & 0.42 & 1.04 & 0.23 & 1.62 \\
\hline Grand average & & 2032 & 1701 & 1.43 & 1.23 & 1.39 & 2.46 & 0.81 & 5.52 & 3.30 & 2.92 & 1.95 & 1.36 \\
\hline
\end{tabular}

Data source: Smith and Srivastava (1986).

$A A D(\%)=\frac{100}{N} \Sigma\left|\frac{\chi^{\mathrm{cal}}-\chi^{\exp }}{\chi^{\exp }}\right|$

; $x=$ saturated vapor pressure or liquid molar volume.

saturated vapor pressures and the liquid molar volumes of nonpolar pure fluids. The results from other EOS: PR (Peng and Robinson, 1976), PT (Patel and Teja, 1982), YL (Yu and Lu, 1987) and CCOR (Lin et al., 1983) are also included for comparison. The proposed EOS is shown superior overall to other cubic EOS in the vapor pressure calculations. The calculated saturated liquid molar volumes of the proposed EOS are slightly better than those from the CCOR, while all other EOS (PR, PT and YL) are not as accurate. It is also worth noticing that this proposed EOS calculated the saturated liquid molar volumes with good accuracy even for long chain hydrocarbons. Fig. 8 compares the calculated vapor pressures with experimental data for $n-C_{10}, n-C_{15}$ and $n-C_{19}$. The agreement is excellent for all these long chain hydrocarbon molecules. A graphical presentation for the saturated liquid volumes of $n$-heptadecane from various EOS is shown in Fig. 9 in which the proposed EOS was again found satisfactory. This EOS also represents well the PVT behavior of pure fluids as illustrated in Fig. 10 for oxygen. The second virial coefficients of pure fluids were not included in the regression of our EOS parameters. Our calculated second virial coefficients for nonpolar pure fluids are comparable to those from the PR and SRK (Soave, 1972) EOS. The correlation of our EOS parameters are based on most compounds in Table 2. These correlations can predict well the saturated properties of other pure nonpolar fluids. For example, the absolute average deviations in the predicted vapor pressure and saturated liquid molar volume for methyl cyclopentane are $0.95 \%$ (38 data points) and $0.45 \%$ ( 35 data points), respectively. 


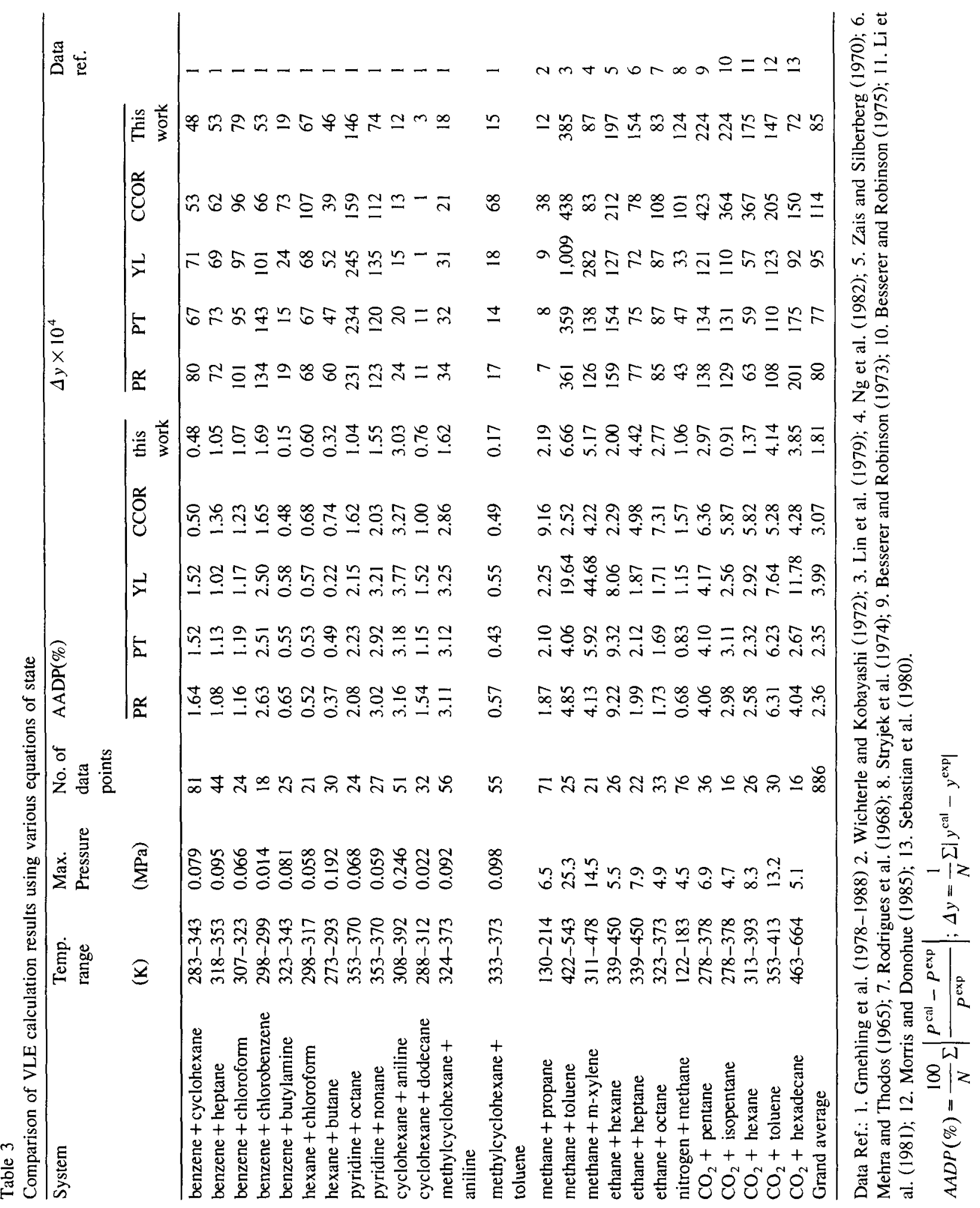




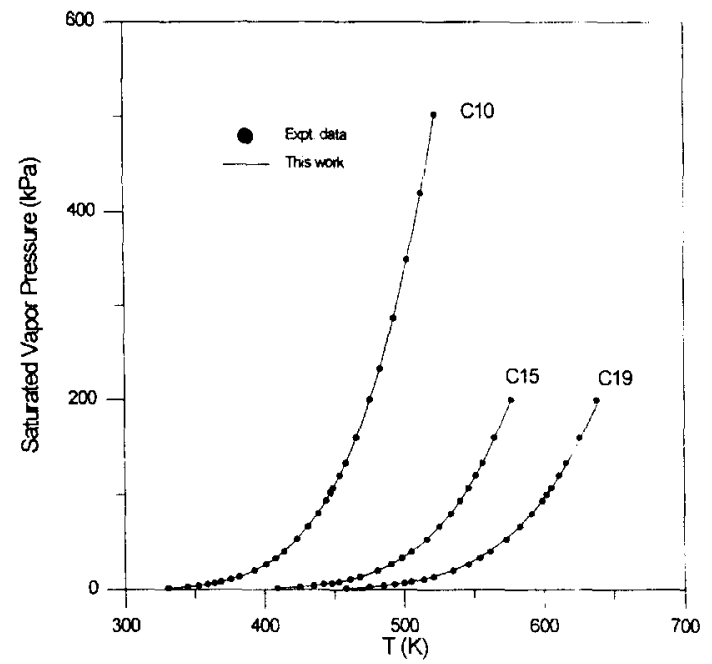

Fig. 8. Comparison of the calculated vapor pressures from this work with the experimental data (Vargaftik. 1975; Smith and Srivastava, 1986).

The VLE calculations for binary nonpolar mixtures from low to high pressures are summarized in Table 3. The calculated results from other commonly-used cubic EOS are also presented in the table for comparison. At least one temperature-independent binary interaction parameter is needed in these mixture calculations. One binary parameter in the mixing rule of $a_{\mathrm{m}}$ (energy parameter) was used for the PR, PT and the YL EOS, while two binary parameters in the mixing rules of both $a_{\mathrm{m}}$ and $b_{\mathrm{m}}$ (energy and volume parameters) were used for the CCOR and our EOS. All the EOS give reasonable

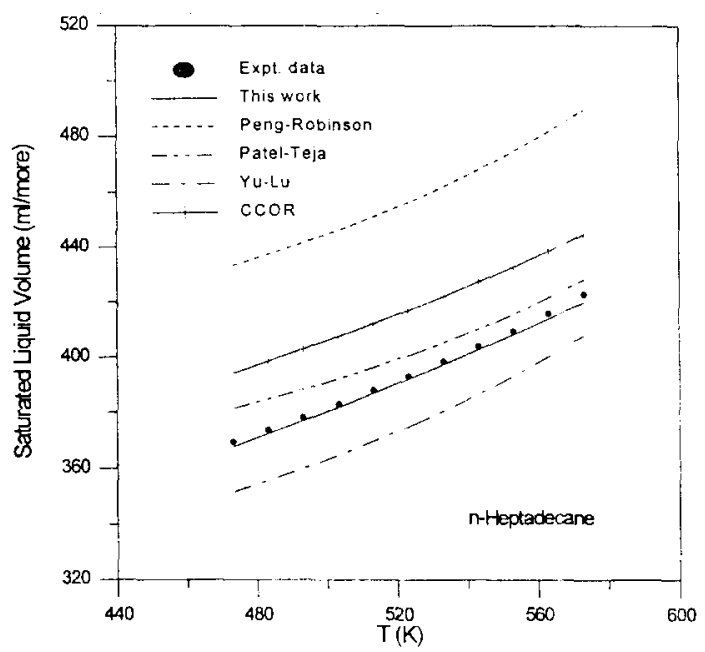

Fig. 9. Comparison of the calculated saturated liquid volumes of $n$-heptadecane from various equations of state (experimental data: Smith and Srivastava, 1986). 


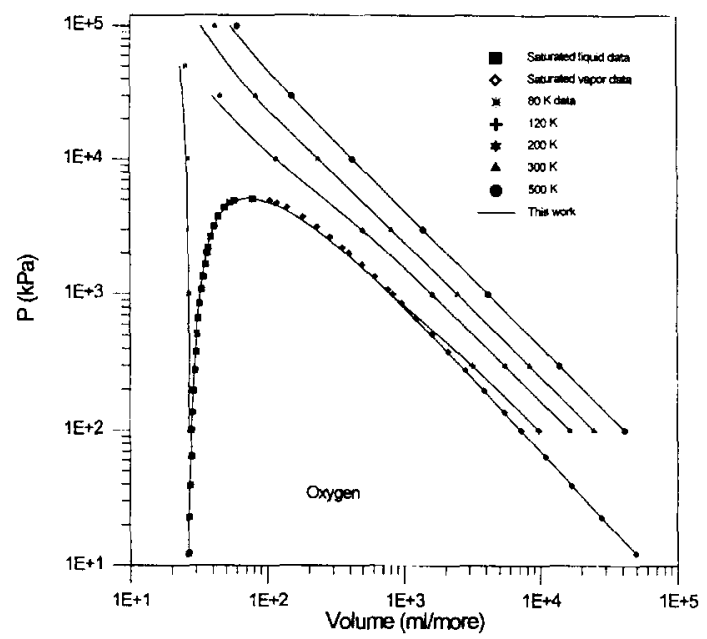

Fig. 10. Comparison of the PVT data for oxygen calculated from this work with the experimental data (Vargaftik, 1975).

results for a majority of mixtures. However, our EOS is slightly better in the calculations of the bubble point pressures and also the vapor phase compositions at low pressures. Table 4 presents a comparison of the calculated saturated liquid volumes from various EOS with the experimental data for binary mixtures at high pressures. The calculations are not sensitive to the binary parameters and, therefore, no parameters were used for any of the EOS. The results demonstrated that the EOS of PT,

Table 4

Comparison of high pressure saturated liquid volumes using various equations of state

\begin{tabular}{|c|c|c|c|c|c|c|c|c|c|}
\hline \multirow[t]{2}{*}{ System } & \multirow{2}{*}{$\begin{array}{l}\text { Temp. } \\
\text { range } \\
(\mathrm{K})\end{array}$} & \multirow{2}{*}{$\begin{array}{l}\text { Max. P } \\
(\mathrm{MPa})\end{array}$} & \multirow{2}{*}{$\begin{array}{l}\text { No. of } \\
\text { data points }\end{array}$} & \multicolumn{5}{|c|}{$\operatorname{AADV}^{\mathrm{L}}(\%)$} & \multirow[t]{2}{*}{ Data ref. } \\
\hline & & & & PR & $\mathrm{PT}$ & YL & SRK & $\begin{array}{l}\text { This } \\
\text { work }\end{array}$ & \\
\hline methane + pentane & $311-411$ & 10.3 & 38 & 2.19 & 1.40 & 0.82 & 11.15 & 1.45 & 1 \\
\hline methane + cyclohexane & $294-444$ & 24.1 & 73 & 4.62 & 1.24 & 2.55 & 7.18 & 0.87 & 2 \\
\hline methane + octane & $298-423$ & 7.1 & 33 & 3.08 & 0.63 & 0.37 & 16.13 & 1.41 & 3 \\
\hline methane + nonane & $223-423$ & 24.3 & 104 & 4.96 & 1.88 & 1.30 & 17.87 & 1.10 & 4 \\
\hline methane + decane & $311-511$ & 25.9 & 101 & 5.40 & 0.94 & 1.61 & 18.45 & 1.31 & 5 \\
\hline ethane + dodecane & $273-373$ & 6.3 & 61 & 4.49 & 1.23 & 1.32 & 17.75 & 3.46 & 6 \\
\hline propane + decane & $277-511$ & 4.1 & 43 & 7.76 & 2.75 & 1.35 & 20.67 & 3.04 & 7 \\
\hline ethylene + dodecane & $283-348$ & 9.1 & 55 & 6.80 & 1.72 & 1.90 & 19.95 & 1.64 & 8 \\
\hline Grand average & & & 508 & 5.17 & 1.52 & 1.52 & 16.38 & 1.66 & \\
\hline
\end{tabular}

Data Ref.: 1. Sage et al. (1942); 2. Reamer et al. (1958); 3. Kohn and Bradish (1964); 4. Shipman and Kohn (1966); 5. Reamer et al. (1942); 6. Reamer and Sage (1966); 7. Lee and Kohn (1969); 8. Ribeiro et al. (1972).

$\operatorname{AADV}^{\mathrm{L}}(\%)=\frac{100}{N} \Sigma\left|\frac{V^{\mathrm{L}, \mathrm{cal}}-V^{\mathrm{L}, \exp }}{V^{\mathrm{L}, \exp }}\right|$ 


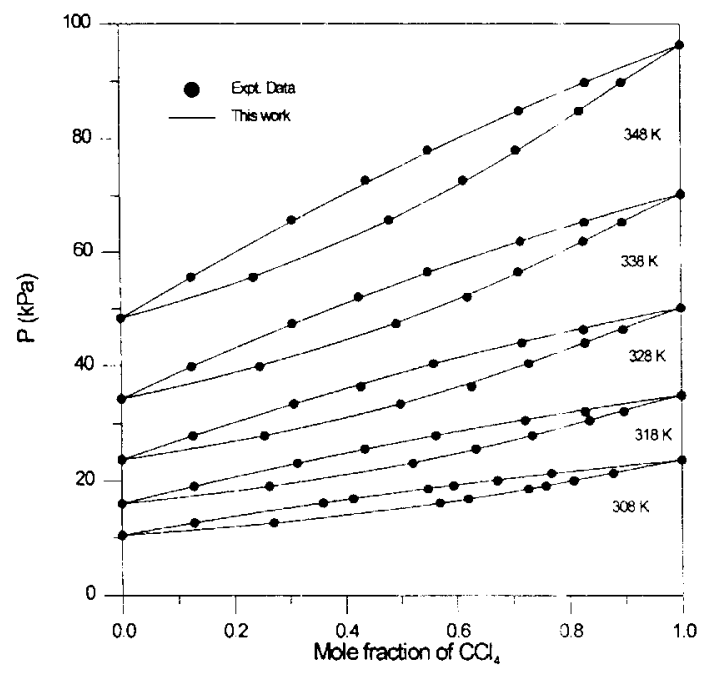

Fig. 11. Comparison of the calculated VLE results of this work with the experimental data (Gmehling et al., 1978-1988) for the binary mixture of carbon tetrachloride $+2,2,4$-trimethylpentane at various temperatures.

YL and this work give comparably good predictions while the PR and the SRK EOS show larger deviations. Graphical illustrations of the VLE calculations with the proposed EOS are presented in Figs. 11-14. Fig. 11 shows the results for the binary mixtures of carbon tetrachloride $+2,2,4$-trimethylpentane. Good agreement with the experimental data was obtained over wide ranges of temperature and pressure. Fig. 12 shows the calculations for the mixture of cyclohexane and dodecane. These components are significantly different in their molecular shapes and sizes. Dodecane is also much less volatile. Nevertheless, excellent results were obtained for this nonideal mixture at various tempera-

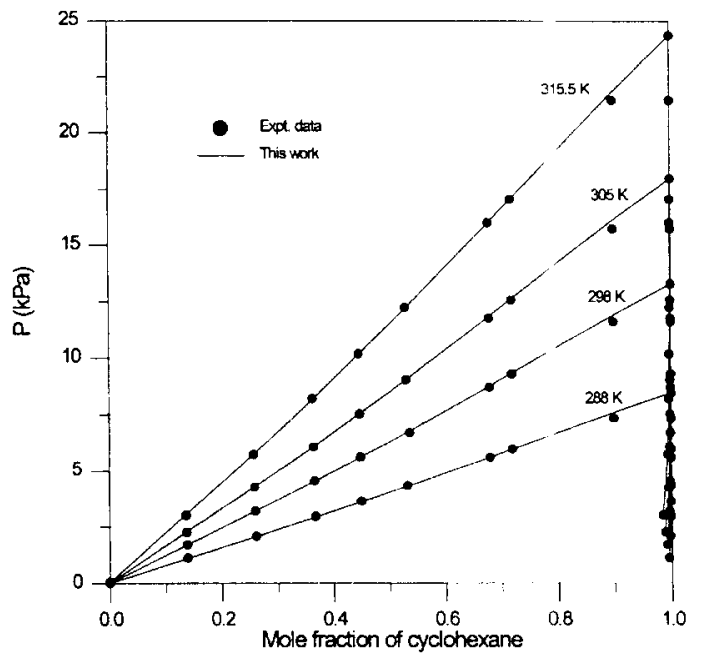

Fig. 12. Comparison of the calculated VLE results of this work with the experimental data (Gmehling et al.. 1978-1988) for the binary mixture of cyclohexane $+n$-dodecane at various temperatures. 


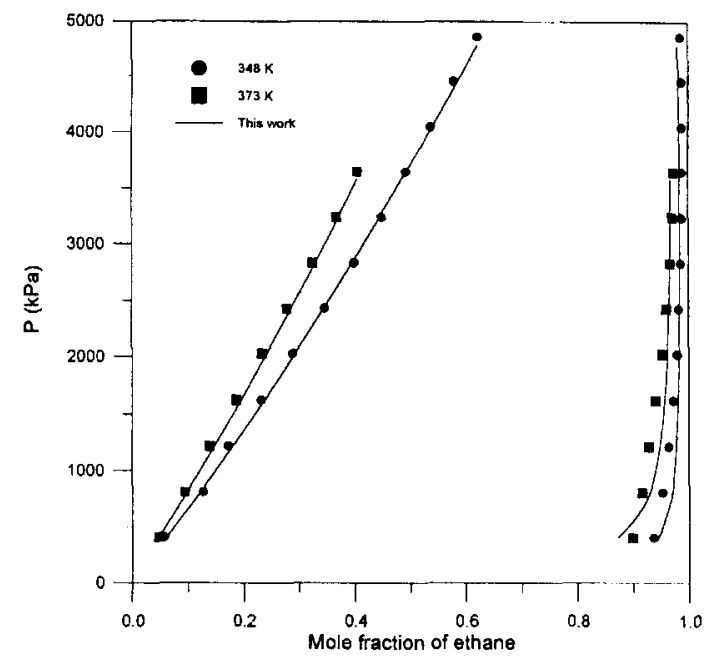

Fig. 13. Comparison of the calculated VLE results of this work with the experimental data (Rodrigues et al., 1968) for the binary mixture of ethane and $\mathrm{n}$-octane at high pressures.

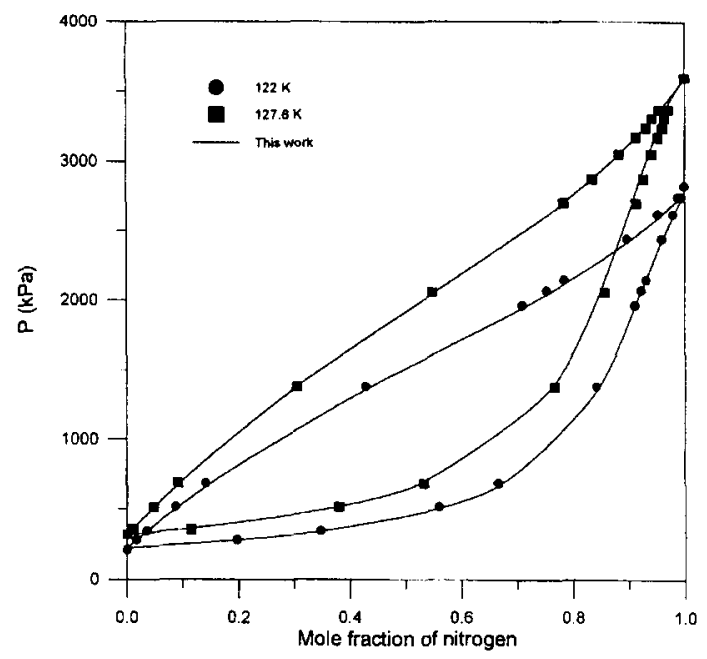

Fig. 14. Comparison of the calculated VLE results of this work with the experimental data (Stryjek et al., 1974) for the binary mixture of nitrogen and methane at high pressures.

tures. Figs. 13 and 14 show the binary mixtures of ethane + n-octane and nitrogen + methane, respectively, at high pressures. The proposed EOS is accurate to represent the VLE behavior of these mixtures.

\section{Conclusion}

A new cubic EOS is presented which consists of a simplified perturbed hard-body term and a simple attractive term. The repulsive term of this EOS yields satisfactory results of the compressibility factor with the molecular simulation data for the hard molecules from a hard sphere to tangent or 
fused hard chainlike fluids. A simple expression for the attractive contribution was added to complete the EOS in a cubic form. The EOS was applied to real fluids, and generalized correlations of the EOS parameters for nonpolar fluids were presented. Satisfactory results for saturated properties were obtained from this EOS, which showed better accuracy in the calculations of saturated liquid volumes and vapor pressures of nonpolar fluids than some other cubic EOS. This EOS also represents well the VLE of nonpolar fluid mixtures. The accuracy of this EOS is comparable to that from other commonly-used cubic EOS for a diversity of binary mixtures.

\section{List of symbols}

$A, B$ temperature-dependent functions in the EOS

$a, b, c$ parameters in the EOS

$F \quad$ correlation functions in Eqs. (24)-(26)

$f \quad$ fugacity

$K \quad$ parameter in the EOS

$P \quad$ pressure

$Q, Q^{\prime} \quad$ objective function

$R \quad$ gas constant

$T \quad$ temperature

$V \quad$ volume

$z \quad$ compressibility factor

\subsubsection{Greek letters}

$\alpha \quad$ nonspherical factor

$\eta \quad$ packing fraction

$\phi \quad$ fugacity coefficient

$\xi_{c} \quad$ critical compressibility factor

$\Omega$ temperature-independent parameters in the EOS

$\omega$ acentric factor

\subsubsection{Superscripts}

exp experimental data

cal calculated results

L liquid phase

V vapor phase

\subsubsection{Subscripts}

c critical state

r reduced property

\section{Acknowledgements}

The authors are grateful to the National Science Council of the Republic of China (NSC83-0402E002-070) for supporting this research. Valuable comments from Dr. H.M. Lin are deeply appreciated. 


\section{References}

Alejandre, J. and Chapela, G.A., 1987. Molecular dynamics for discontinuous potentials III. Compressibility factors and structure of hard polyatomic fluids, Molec. Phys., 61: 1119-1130.

Amos, M.D. and Jackson, G., 1991. BHS theory and computer simulations of linear heteronuclear triatomic hard-sphere molecules, Molec. Phys., 74: 191-210.

Besserer, G.J. and Robinson, D.B., 1973. Equilibrium phase properties of n-pentane-carbon dioxide system. J. Chem. Eng. Data, 18: 416-419.

Besserer, G.J. and Robinson, D.B., 1975. Equilibrium phase properties of isonpentane-carbon dioxide system. J. Chem. Eng. Data, 20: 93-96.

Boublik, T. and Nezbeda, I., 1986. P-V-T behavior of hard body fluids. Theory and experiment, Coll. Czech. Chem. Commun., 51: 2301-2432.

Boublik, T., Vega, C. and Diaz-Pena, M., 1990. Equation of state of chain molecules, J. Chem. Phys., 93: $730-736$.

Carnahan, N.F. and Starling, K.E., 1969. Equation of state for non-attracting rigid spheres. J. Chem. Phys., 51: $635-636$.

Denlinger, M.A. and Hall, C.K., 1990. Molecular-dynamics simulation results for the pressure of hard-chain fluids, Molec. Phys., 71: 541-559.

Dickman, R. and Hall, C.K., 1988. High density Monte Carlo simulations of chain molecules: Bulk equation of state and density profile near walls, J. Chem. Phys., 89: 3168-3174.

Elliott, J.R. Jr., Suresh, S.J. and Donohue, M.D., 1990. A simple equation of state for nonspherical and associating molecules, Ind. Eng. Chem. Res., 29: 1476-1485.

Gao, J. and Weiner, J.H., 1989. Contribution of covalent bond force to pressure in polymer melts, J. Chem. Phys., 91: 3168-3173.

Gmehling, J., Onken, U., Arlt, W., Grenzheuser, P., Weidlich, U., Kolbe, B. and Rarey-Nies, J., 1978-1988. Vapor-liquid equilibrium data collection. DECHEMA, Frankfurt.

Kohn, J.P. and Bradish, W.F., 1964. Multiphase and volumetric equilibria of the methane-n-octane system at temperatures between $-110^{\circ} \mathrm{C}$ and $150^{\circ} \mathrm{C}$. J. Chem. Eng. Data, 9: 5-8.

Lee, K.H. and Kohn, J.P., 1969. Heterogeneous phase equilibrium in the ethane-n-dodecane system. J. Chem. Eng. Data, 14: 292-295.

Li, Y.H., Dillard, K.H. and Robinson, R.L., 1981. Vapor-liquid phase equilibrium for carbon dioxide-n-hexane at 40,80 and $120^{\circ} \mathrm{C}$. J. Chem. Eng. Data, 26: 53-55.

Lin, H.M., Kim, H., Guo, T.M. and Chao, K.C., 1983. Cubic chain-of-rotators equation of state and VLE calculations, Fluid Phase Equilibria, 13: 143-152.

Lin, H.M., Sebastian, H.M., Simnick, J.J. and Chao, K.C., 1979. Gas-liquid equilibrium in binary mixtures of methane with n-decane, benzene and toluene. J. Chem. Eng. Data, 24: 146-149.

Mehra, V.S. and Thodos, G., 1965. Vapor-liquid equilibrium in the ethane-n-heptane System. J. Chem. Eng. Data, 10: 211-214.

Morris, W.O. and Donohue, M.D., 1985. Vapor-liquid equilibria in mixtures containing carbon dioxide, toluene, and 1-methylnaphthalene. J. Chem. Eng. Data, 30: 259-263.

$\mathrm{Ng}$, H.J., Huang, S.S. and Robinson, D.B., 1982. Equilibrium phase properties of selected m-xylene binary systems. m-xylene-methane and m-xylene-carbon dioxide. J. Chem. Eng. Data, 27: 119-122.

Patel, N.C. and Teja, A.S., 1982. A new cubic equation of state for fluids and fluids mixtures, Chem. Eng. Sci., 37: 463-473.

Peng, D.Y. and Robinson, D.B., 1976. A new two-constant equation of state, Ind. Eng. Chem. Fund., $15: 59-64$.

Reamer, H.H., Sage, B.H. and Lacey, W.N., 1942. Phase equilibria in hydrocarbon systems. Methane-decane system. Ind. Eng. Chem., 34: 1526-1531.

Reamer, H.H., Sage, B.H. and Lacey, W.N., 1958. Phase equilibria in hydrocarbon systems. volumetric and phase behavior of the methane-cyclohexane system. J. Chem. Eng. Data, 3: 240-245.

Reamer, H.H. and Sage, B.H., 1966. Phase equilibria in hydrocarbon systems. Volumetric and phase behavior of the propane-n-decane system. J. Chem. Eng. Data, 11: 17-24.

Ribeiro, J.V., Susu, A.A. and Kohn, J.P., 1972. Heterogeneous phase equilibrium in ethylene-n-dodecane system. J. Chem. Eng. Data, 17: 279-280. 
Rodrigues, A.B.J., McCaffery, D.S. and Kohn, J.P., 1968. Heterogeneous phase and volumetric equilibrium in the ethane-n-octane system. J, Chem. Eng. Data, 13: 164-168.

Sage, B.H., Reamer, H.H., Olds, R.H. and Lacey, W.N., 1942. Phase equilibria in hydrocarbon systems. Volumetric and phase behavior of methane-n-pentane system. Ind. Eng. Chem., 34: 1108-1117.

Sebastian, H.M., Simnick, J.J., Lin, H.M. and Chao, K.C., 1980. Vapor-liquid equilibrium in binary mixtures of carbon dioxide $+n$-decane and carbon dioxide $+n$-hexadecane. J. Chem. Eng. Data, 25: 138-140.

Shah, V.M., Bienkowski, P.R. and Cochran, H.D., 1994. Generalized quartic equation of state for pure nonpolar fluids, AIChE J., 40: 152-159.

Shipman, L.M. and Kohn, J.P., 1966. Heterogeneous phase and volumetric equilibrium in the methane-n-nonane system. J. Chem. Eng. Data, 11: 176-180.

Smith, B.D. and Srivastava, R., 1986. Thermodynamic Data for Pure Compounds. Elsevier, Amsterdam.

Soave, G., 1972. Equilibrium constants from a modified Redlich-Kwong equation of state. Chem. Eng. Sci., 27: $1197-1203$

Soave, G., 1990. A non-cubic equation of state for PVT and phase equilibrium calculations (pure compounds). Fluid Phase Equilibria, 56: 39-57.

Song, Y. and Mason, E.A., 1990. Equation of state for a fluid of hard convex bodies in any number of dimensions, Phys. Rev., A41: $3121-3124$.

Stryjek, R., Chappelear, P.S. and Kobayashi, R., 1974. Low temperature vapor-liquid equilibria of nitrogen-methane system. J. Chem. Eng. Data, 19: 334-339.

Thomas, A. and Donohue, M.D., 1993. Equation of state for chain molecules using a site-interaction model: theory, simulation, and correlation of experimental data, Ind. Eng. Chem. Res., 32: 2093-2104.

Vargaftik, N.B., 1975. Tables on the Thermophysical Properties of Liquids and Gases. Hemisphere, Washington, DC.

Walsh, J.M. and Gubbins, K.E., 1990. A modified thermodynamic perturbation theory equation for molecules with fused hard sphere cores, J. Phys. Chem., 94: 5115-5120.

Wang, L.S. and Guo, T.M., 1993. A cubic simplified perturbed hard-chain equation of state for fluids with chainlike molecules, Can. J. Chem. Eng., 71: 591-604.

Wichterle, I. and Kobayashi, R., 1972. Vapor-liquid equilibrium of methane-propane system at low temperatures and high pressures. J. Chem. Eng. Data, 17: 4-9.

Yu, J.M. and Lu, B.C.Y., 1987. A three-parameter cubic equation of state for asymmetric mixture density calculations, Fluid Phase Equilibria, 34: 1-19.

Zais, E.J. and Silberberg, I.H., 1970. Vapor-liquid equilibrium in the ethane-n-hexane system. J. Chem. Eng. Data, 15: 253-256. 